\title{
Parental ages and levels of DNA methylation in the newborn are correlated
}

\author{
Ronald M Adkins ${ }^{*}$, Fridtjof Thomas ${ }^{2}$, Frances A Tylavsky ${ }^{2}$ and Julia Krushkal ${ }^{2}$
}

\begin{abstract}
Background: Changes in DNA methylation patterns with age frequently have been observed and implicated in the normal aging process and its associated increasing risk of disease, particularly cancer. Additionally, the offspring of older parents are at significantly increased risk of cancer, diabetes, and neurodevelopmental disorders. Only a proportion of these increased risks among the children of older parents can be attributed to nondisjunction and chromosomal rearrangements.
\end{abstract}

Results: Using a genome-wide survey of 27,578 CpG dinucleotides in a cohort of 168 newborns, we examined the relationship between DNA methylation in newborns and a variety of parental and newborn traits. We found that methylation levels of 144 CpGs belonging to 142 genes were significantly correlated with maternal age. A weaker correlation was observed with paternal age. Among these genes, processes related to cancer were overrepresented, as were functions related to neurological regulation, glucose/carbohydrate metabolism, nucleocytoplasmic transport, and transcriptional regulation. CpGs exhibiting gender differences in methylation were overwhelmingly located on the X chromosome, although a small subset of autosomal CpGs were found in genes previously shown to exhibit gender-specific differences in methylation levels.

Conclusions: These results indicate that there are differences in $\mathrm{CpG}$ methylation levels at birth that are related to parental age and that could influence disease risk in childhood and throughout life.

\section{Background}

DNA methylation is a normal, heritable epigenetic modification that down-regulates the expression of approximately $1 / 3$ of human genes [1-3] and is key to the allele-specific imprinting of genes [4]. DNA methylation also plays an important role in disease. For example, overall DNA hypomethylation accompanied by genespecific hypermethylation is a hallmark of cancer [5]. Additionally, shifts in DNA methylation patterns appear to be involved in the normal aging process and increase in disease susceptibility [6]. Indeed, there is ample evidence for characteristic changes in the patterns of DNA methylation with age.

The earliest data supporting progressive changes in DNA methylation patterns with age came from global studies of blood that demonstrated lower levels of methylation in older individuals [7] and greater

\footnotetext{
* Correspondence: ronald.m.adkins@gmail.com

'Department of Pediatrics, University of Tennessee Health Science Center, Memphis, TN 38103, USA

Full list of author information is available at the end of the article
}

differences in methylation in older monozygotic twins [8]. Christensen et al. [9] examined 217 tissues sampled from a range of non-diseased solid tissues and blood. In solid tissues the general trend was towards methylation increasing with age in $\mathrm{CpG}$ islands and decreasing outside of islands. However, in 85 blood samples the predominant pattern was a decrease in DNA methylation with age that was independent of CpG island status. In an analysis of $280 \mathrm{CpGs}$ measured from whole blood from 46 monozygotic and dizygotic twin pairs and 96 singleton adults, Boks et al. [10] found both increases (60\%) and decreases in methylation with age among the 100 most significant results. As previously observed, levels of methylation for X-linked loci were higher among females. Heritability estimates for methylation levels for 25 significant $\left(\mathrm{p} \leq 10^{-3}\right)$ CpGs ranged from $0.57-0.94$. Similarly, in whole blood samples from 93 females using Illumina's Humanmethylation27 BeadChip (the same microarray as used in this study) Rakyan et al. [11] found 213 CpGs whose methylation levels positively correlated with age ("hyper-aDMRs") and 147 that 
negatively correlated. In a replication cohort of 20 females and 5 males, 60\% (131) of CpGs whose methylation increased with age replicated in $\mathrm{CD} 14^{+}$monocytes and $\mathrm{CD}^{+}{ }^{+} \mathrm{T}$-cells, while the CpGs whose methylation decreased replicated in the T-cells but not monocytes. Rakyan et al. suggested that the lack of replication in the monocytes was due to changes in cell composition in blood with age. Only the hyper-aDMRs were studied in buccal cells from ten individuals, where they showed high replication. A larger study [12] ( $\mathrm{N}=197,21$ - 55 years old) of DNA isolated from the saliva of alcohol abusers using the same Illumina array as the one reported here found 85 CpGs significantly correlated (including 29 negatively) with age after Bonferroni correction. In another study using the same array for blood from 261 postmenopausal women, Teschendorff et al. [13] found significant hypermethylation of 226 CpGs and hypomethylation of $363 \mathrm{CpGs}$ with age. Replication results were not presented for all significant CpGs, but focusing on only those mapped to polycomb group proteins the 64 genes with hypermethylated sites replicated in other tissues and individuals, while the 11 hypomethylated genes generally did not. Once again, this could be due to changes in blood cell composition with age.

The studies summarized above investigated the relationship between age and DNA methylation levels in a cross-sectional sample of individuals at a fixed point in time. Studies that have examined changes in DNA methylation levels in the same individuals over time have revealed similar results. Bollati et al. [14] studied levels of DNA methylation in Alu and LINE-1 repetitive elements in serial blood samples taken up to eight years apart from 718 elderly Boston individuals (55 - 92 years old). They found a significant correlation between age and DNA methylation in both types of elements and significant declines in methylation in $A l u$ elements in the same individual over time. Similarly, Bjornsson et al. [15] studied changes in DNA methylation in blood in the same individuals over a span of greater than 10 years. Although results were not presented for all families or as summaries across all families, the 50 largest changes in blood mostly appeared to be decreases both inside and outside of $\mathrm{CpG}$ islands. After excluding one family that exhibited unusually consistent changes in DNA methylation over time, Bjornsson et al. estimated a heritability for DNA methylation change over time of 0.743 .

There is typically a very high correlation between the ages and social/environmental exposures of parents that makes it difficult to disentangle the relative roles of maternal and paternal ages in adverse outcomes in their offspring. Generally, very large epidemiological studies are required that include a statistically meaningful subset of parents with discordances in age. Based on such studies, both maternal and paternal age correlate with elevated risks of complications during pregnancy and complex disorders in the offspring, such as intrauterine growth retardation, altered placental morphology, preeclampsia, and preterm labor [16]. Although the connection is controversial in some cases, the offspring of older parents have increased risks of various disorders, such as type 1 diabetes [17], obsessive-compulsive disorders, autism [18], schizophrenia [19], bipolar disorder [20], stuttering [21], hematologic cancers [22], and the more common childhood cancers [23-29]. Furthermore, daughters of older women have an increased risk of breast cancer [30], and the sons of older fathers have an increased risk of prostate cancer [31]. The role of epigenetics in these associations is only beginning to be explored. The only recent recognition of a possibly large role of epigenetics may be partially attributed to assumptions that each generation begins with a "renewed" epigenome. After fertilization the maternal and paternal genomes undergo a rapid demethylation of most of the genome followed by remethylation. Naively, this suggests that each generation begins with a refreshed epigenome that, barring gross environmental insults or altered dietary intakes [32], is relatively immune to effects from the previous generation $[33,34]$. Here we report the results of genome-wide DNA methylation profiling of 168 newborns that indicates that there is a general trend towards hypomethylation of $\mathrm{CpG}$ islands in newborn blood cells with increasing parental age. It is possible that this trend provides a partial explanation for the association between increased parental age and adverse outcomes in offspring.

\section{Results}

Final characteristics of the data set and population

Across all 168 newborns, two CpGs exceeded a median detection $\mathrm{p}$ value of $10^{-6}$ and were excluded from analysis. The final data set was comprised of $27,576 \mathrm{CpG}$ dinucleotides. An average of $184 \pm 498$ (range 0 - 2,989) CpGs per newborn exceeded the threshold detection $\mathrm{p}$ value of $\leq 10^{-3}$, and those specific CpGs within individual newborns were dropped from analyses.

The distribution of maternal ages was skewed towards younger mothers, with $41 \%(\mathrm{~N}=69)$ being younger than 26 and the proportion diminishing with increasing age (Table 1 ; 26-30 yrs, 33\%, $\mathrm{N}=55$; 31-35 yrs, 20\%, $\mathrm{N}=34$; 36-39 yrs, 6\%, $\mathrm{N}=10$ ). Most mothers were classified as normal weight or overweight at the time of recruitment according to body mass index (BMI), with 5.4\% $(\mathrm{N}=9)$ mothers being underweight (BMI < 18.5), $46 \%(\mathrm{~N}=77)$ normal (BMI = 18.5 - 24.9), $20 \%(\mathrm{~N}=34)$ overweight $(\mathrm{BMI}=25-29.9)$, and $29 \%(\mathrm{~N}=48)$ obese (BMI > 29.9). The majority of newborns were born at term ( $\geq 37$ weeks; $N=158$ ), while seven were near-term 
Table 1 Characteristics of the participants $(n=168)$

\begin{tabular}{lll}
\hline Variable & Mean (SD) & Range \\
\hline Race (Maternal) & 92 & \\
African-American (N) & 70 & \\
Caucasian (N) & 6 & \\
Other, Multiracial (N) & & $18-39$ \\
Mothers & $27.0(5.1)$ & $0-5$ \\
Age (years) & $1.1(1.2)$ & $15.3-54.9$ \\
Parity & $27.0(7.3)$ & $18-50$ \\
BMl (Kg/m ${ }^{2}$ ) & 15 & $32-41$ \\
Smoke during pregnancy (N) & $31.6(7.1)$ & \\
Paternal age (N = 89) & $39.0(1.3)$ & $1.2-5.0$ \\
Newborns & 80 & \\
Gestational age (weeks) & 3.3 & \\
Female (N) &
\end{tabular}

(35 - 36 weeks) and three were preterm (32 - 33 weeks). Birth weights ranged from 1.2 to $5 \mathrm{Kg}$, with the lower end of the range primarily due to the small number of preterm newborns. There were slightly more male $(\mathrm{N}=$ $88,52 \%)$ than female newborns. There was no difference in the average age of mothers giving birth to female or male newborns ( $t$ test, $\mathrm{p}=0.91$ ). The age was reported for 89 fathers, which averaged 31.6 years. Unlike the maternal ages, the distribution of paternal ages was normally distributed (Shapiro-Wilk W test, $\mathrm{p}=0.74 ; 18$ - 25 years, $20 \%, \mathrm{~N}=18 ; 26$ - 30 years, $21 \%, \mathrm{~N}=19 ; 31$ - 35 years, $29 \%, N=26 ; 36$ - 40 years; $21 \%, N=19 ; 41$ - 50 years, $8 \%, \mathrm{~N}=7$ ).

\section{Relationship between newborn CpG methylation levels and maternal/newborn variables}

No CpG displayed a significant relationship between methylation level and gestational age, parity, maternal BMI, birth weight, or to self-declared smoking during pregnancy. Across all chromosomes, the levels of methylation at $144 \mathrm{CpG}$ loci located in 140 autosomal (2 each in ARFGAP3 and MEA1) and $2 \mathrm{X}$-linked genes exhibited genome-wide significant correlations with maternal age (Additional File 1, Table S1). Relative to other loci, imprinted genes generally maintain a stringent pattern of allele-specific methylation in a parent of origin manner. Nevertheless, a single $\mathrm{CpG}$ in each of two imprinted genes (KCNQ1 and DIRAS3) were the third- and eighteen-ranked sites (rho $=-0.41$ and -0.40 , respectively) correlated with maternal age. The level of methylation at all but 9 of the 144 genome-wide significant CpGs was negatively correlated with maternal age. With the exception of six genes, at least two CpGs are interrogated in each of the 142 genes containing genome-wide significant $\mathrm{CpG}$ probes. To determine if the pattern we observe for the genome-wide significant $\mathrm{CpGs}$ is reflected by another $\mathrm{CpG}$ in the same gene, we examined the direction and significance of the Spearman rho for the CpG with the next lowest p value. Of the 136 genes represented by multiple probes, 95 of the secondranked $\mathrm{CpGs}$ exhibited a correlation in the same direction as the top-ranked $\mathrm{CpG}$, of which 53 were significant at a nominal $\mathrm{p}$ value $\leq 0.05$ (Additional File 2, Table S2). The levels of methylation of these 53 pairs of CpGs are significantly correlated (Spearman's rho = $\left.0.26-0.82, \mathrm{p}=6.3 \times 10^{-4}-2.1 \times 10^{-42}\right)$, except the pair in the GORASP1 gene (rho $=0.07, \mathrm{p}=0.39$ ).

Of the $144 \mathrm{CpG}$ s exhibiting a significant correlation with maternal age, all but $8(5.6 \%)$ are located in CpG islands. By contrast, 7,568 (27.45\%) of the CpGs in the total data set are outside of a $\mathrm{CpG}$ island. If there were no relationship between the $\mathrm{CpG}$ island status of each probe and its correlation with maternal age, we would expect 39-40 of the significantly correlated CpGs to be outside of an island. Therefore, there is a significant (binomial test, $\mathrm{p}=1.6 \times 10^{-11}$ ) over-representation of $\mathrm{CpG}$ island sites among the significant correlations. Of the nine significantly positive correlations between maternal age and newborn CpG methylation, four are outside of a CpG island. Indeed, overall there appears to be a different relationship between maternal age and levels of methylation of CpGs located inside and outside of CpG islands (Figure 1). Outside of CpG islands, the distribution of Spearman rho statistics is slightly shifted towards positive correlations (median $=0.02$ ), while inside CpG islands the correlations are predominantly negative (median $=-0.1$ ).

Of the 834 CpG loci (501 genes) whose methylation levels are significantly associated with newborn gender,

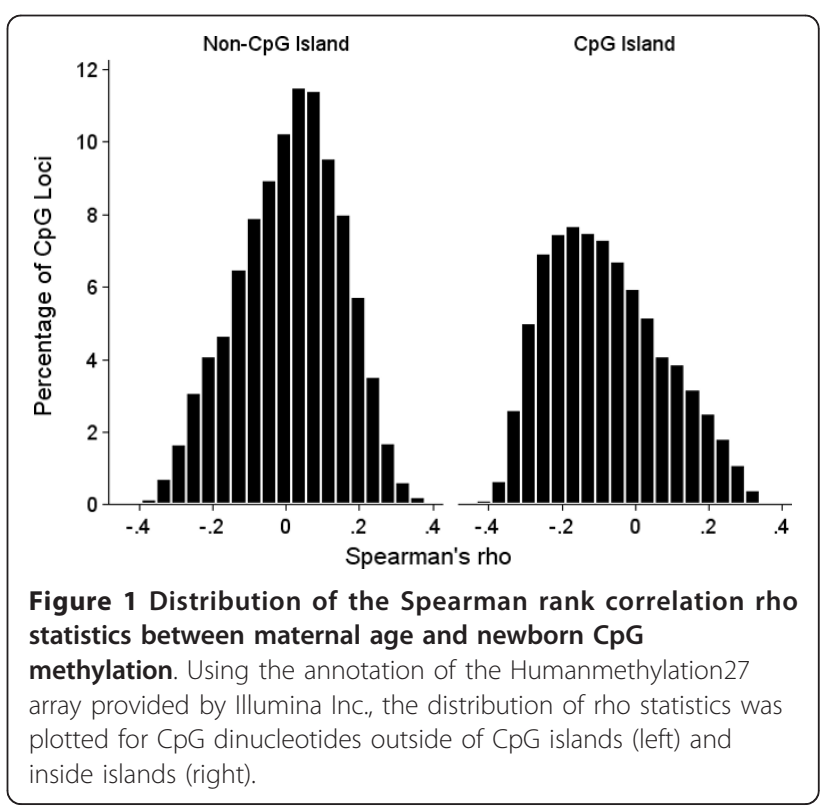


all but 16 (14 genes) are located on the X chromosome, and these associations are presumably due to a systematic difference between males and females in the average level of DNA methylation on the X [35]. Six (C6orf68, TLE1, GLUD1, NUPL1, FLJ20582, FLJ43276) of the fourteen autosomal loci exhibiting gender-specific differences in methylation levels were also among the eleven loci found to exhibit genome-wide significant gender differences in adult saliva DNA based on the same Illumina array in a study by Liu et al. [12]. Additionally, and consistent with our observation, Philibert et al. [36] demonstrated higher methylation of the SLC6A4 gene, posited to be important in several psychiatric and behavioral traits, in adult females. Combined, these results indicate that these gender-specific differences in methylation of a small number of autosomal genes are replicable, present in multiple tissues, and established by the time of birth. Other than zona pellucida-binding protein 2 (ZPBP2), none of the 14 autosomal genes has an obvious function related to gender. The gender difference in X chromosome methylation could confound the ability to detect a relationship to maternal age in analyses that include both genders. When analyses were restricted to individual genders, the DNA methylation level of one X-linked probe (cg08800033 at CETN2) was genome-wide significant in male newborns, and none were significant in females. If a less stringent Bonferroni cut-off $\left(\mathrm{p}=4.6 \times 10^{-5}\right)$ is employed based on the 1,085 CpGs located on the $\mathrm{X}$, then three X-linked loci (cg08800033 at CETN2, cg14570389 at RAP2C, and cg17880859 at RBBP7) in males and one (cg06350107 at OTC) in females is significant. In light of these results and the expected decrease in power to detect a trend in single genders, it is possible that the levels of methylation of a small subset of X-linked CpGs exhibit a correlation with maternal age in a gender-specific manner. However, the support for this is weak, and we did not further examine gender-specific patterns for X-linked CpGs with respect to maternal age.

\section{Replication of maternal age correlation by pyrosequencing}

For 92 newborn DNA samples, the correlation between maternal age and DNA methylation was reassessed using pyrosequencing for three CpGs. These CpGs were chosen based on their different levels of methylation and the correlation of the methylation levels with maternal age. Those three sites and their levels of methylation and correlation with maternal age based on Illumina data were $\operatorname{cg} 09118625(47-82 \%$, rho $=-0.38$, $\mathrm{p}=-.0002), \operatorname{cg} 18984151(10-25 \%$, rho $=-0.34, \mathrm{p}=$ $0.001)$, and $\operatorname{cg} 21576698(3-23 \%$, rho $=-0.31, \mathrm{p}=$ $0.003)$. Based on pyrosequencing, the correlations with maternal age were replicated $(\operatorname{cg} 09118625$, rho $=-0.27$, $\mathrm{p}=0.009 ; \mathrm{cg} 18984151$, rho $=-0.28, \mathrm{p}=0.007$; cg21576698, rho $\left.=-0.42, \mathrm{p}=3.6 \times 10^{-5}\right)$. However, while the inferred levels of methylation of cg18984151 from pyrosequencing $(12-23 \%)$ were very similar to and highly correlated with the values from the array (rho $\left.=0.75, \mathrm{p}=4.6 \times 10^{-18}\right)$, the correlations for the other sites were strong but the inferred methylation levels shifted downwards (cg09118625, $39-48 \%$, rho = $0.73, \mathrm{p}=2.4 \times 10^{-16} ; \mathrm{cg} 21576698,0-1.4 \%$, rho $=0.28$, $\mathrm{p}=0.008)$. Altogether these results indicate that the correlation between maternal age and newborn DNA methylation is replicable, but that the absolute methylation values derived from the Humanmethylation 27 array for some sites may be biased indicators of the true values and may overestimate the true level of inter-individual variation in methylation levels.

\section{Relative strength of the correlation of maternal vs. paternal age with newborn CpG methylation}

The ages of the mothers and fathers were highly correlated in our sample (Figure 2; Spearman rho $=0.75, \mathrm{p}=$ $2 \times 10^{-17}$ ). As a consequence, it would be extremely difficult to distinguish between the effects of maternal and paternal age. This is particularly the case given that there are only 31 cases of an age difference $\geq 5$ years and 7 cases of an age difference $\geq 10$ years, providing little statistical power to examine these subsets as a unique category. The relative strength of the relationship between CpG methylation and parental ages can be qualitatively assessed by examining the relative magnitude of the correlation between methylation levels and the ages of the 89 mothers and fathers for which we have the ages of both. With only 89 observations, the power to achieve genome-wide significance is reduced. For paternal age, there are 6 correlations with a $p$ value

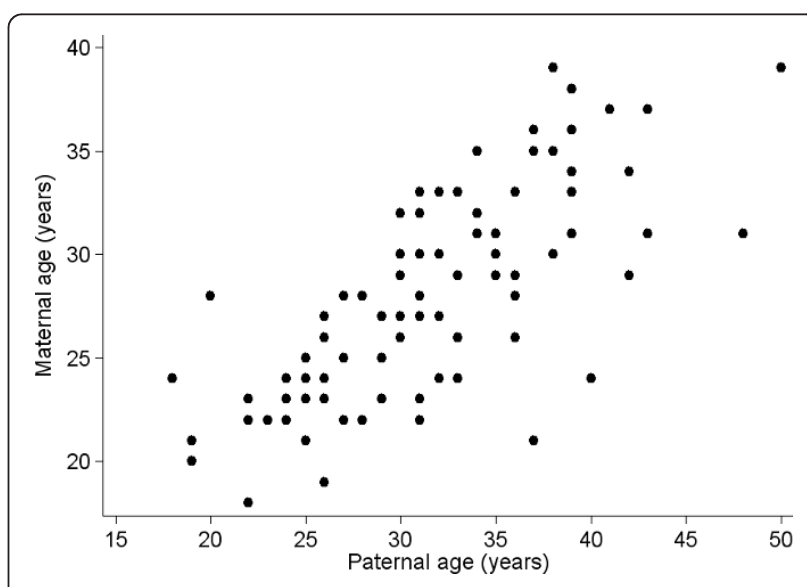

Figure 2 Scatterplot comparison of maternal and paternal ages among 89 newborns for which the age of both parents was known. 
$\leq 10^{-4}\left(84\right.$ at $\left.\mathrm{p} \leq 10^{-3}\right)$, while for maternal age there is one $\mathrm{CpG}$ significant at the genome-wide level (cg00231644 in TUBB2A, $\left.\mathrm{p}=1.54 \times 10^{-6}\right), 7$ additional CpGs with $\mathrm{p} \leq 10^{-5}$, and a total of 106 with $\mathrm{p} \leq 10^{-4}$. Therefore, it appears that the relationship of newborn CpG methylation with maternal age is stronger and involves a larger number of sites than does that with paternal age.

\section{Role of Population Stratification or Sequence Polymorphism}

We have previously demonstrated extensive differences in DNA methylation levels between African-Americans and Caucasians [37]. Therefore, our observed correlations between maternal age and newborn DNA methylation levels could be confounded by these racial differences, particularly given that the African-American mothers are generally younger than the Caucasian mothers $(\mathrm{p}=0.0001$, Wilcoxon rank-sum test). Based on plots of the first two principal components using genome-wide single nucleotide polymorphism (SNP) data from a subset of newborns (60 African-American, 71 Caucasian; Figure 3), two rather distinct clusters of individuals defined by both principal components are clear. One cluster is composed of 57 maternally-declared African-American and 3 Caucasian newborns, and the other contains 6 African-American and 65 Caucasian newborns. As expected based on the significant difference in maternal ages between the two races, the first genetic principal component is significantly correlated with maternal age ( $r$ ho $=-0.28, \mathrm{p}=0.0001$ ), reinforcing the possibility for confounding of racial and maternal age effects. However, within each of the two clusters, the correlation between the first principal component

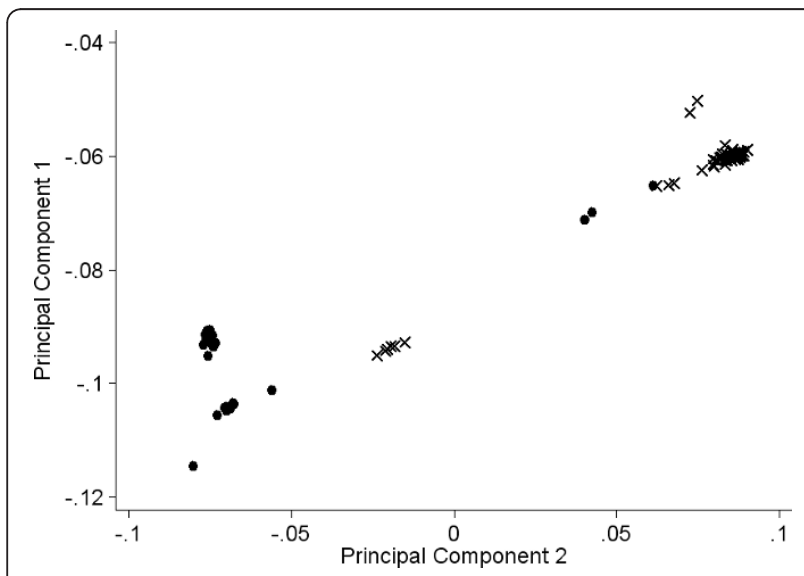

Figure 3 Scatterplot of newborns along the first and second principal components calculated from genome-wide single nucleotide polymorphism data. Maternally-declared Caucasian newborns are represented by circles and African-American newborns by X's. and maternal age was not significant $(\mathrm{p}>0.2)$, suggesting that analyses within these clusters of modest genetic homogeneity would be much less subject to confounding of racial, genetic, and maternal age effects.

Within each cluster defined by genetic principal components, correlation analysis was performed between maternal age and DNA methylation at each CpG. Given the reduction in the sample size within each cluster to less than half the total sample size, it is not surprising that only one CpG achieves a genomewide significant correlation with maternal age. Nevertheless, for the purposes of evaluating the possibility of confounding between maternal age and race, it is more relevant to compare the trends between the genetically distinct clusters of newborns. A correlation analysis of the Spearman rho statistics between the two clusters is highly significant $\left(\mathrm{rho}=0.39, \mathrm{p}<10^{-14}\right.$; using newborns declared as only one race, $\mathrm{N}=162$, rho $=0.41, \mathrm{p}<10^{-}$ ${ }^{14}$ ), indicating that similar trends exist in both groups. Restricting attention to rho statistics with a magnitude $\geq$ 0.2 (roughly $\mathrm{p} \leq 0.1$ ), indicating a relatively strong trend, there are 2,584 CpGs which achieve this level in both groups. Of these, only 175 (7\%) have rho statistics with opposite signs, meaning strong trends in the opposite directions. Overall, these results indicate that correlations between maternal age and newborn CpG methylation are similar in groups defined by race or genetic principal components and that confounding by racial differences in DNA methylation levels or average maternal age is not a major influence.

Although overall racial differences in DNA methylation levels do not appear to be confounding our observation of correlations between maternal age and newborn DNA methylation, a more subtle confounding could arise from the presence of sequence polymorphisms, particularly SNPs, within the regions targeted by probes on the Humanmethylation 27 array whose allele frequencies differ between the races and which could affect the hybridization of DNA to the array. Using the UCSC Genome Browser http://genome.ucsc.edu we examined a window of 50 nucleotides upstream and downstream of each of the 144 genomewide significant CpGs correlated with maternal age. Of the 144 significant CpGs, 60 are within 50 nucleotides of at least one polymorphism listed within dbSNP http://www.ncbi.nlm. nih.gov/SNP/. Therefore, up to $42 \%$ of our genomewide significant correlations with maternal age could be artifacts caused by known polymorphisms in the human genome. Given that we used a rather liberal criterion for defining potential sequence effects and did not directly determine if the probes on the Illumina arrays physically overlap these polymorphisms, the number of potential artifacts due to altered hybridization is undoubtedly lower than $42 \%$. 
Functional Clusters and Disease Processes

Among the 1,494 CpGs correlated with maternal age at a false discovery rate of $6.69 \times 10^{-4}$ (see Methods), 1,423 genes are represented, including 12 imprinted loci (KCNQ1, PRIM2, PLAGL1, GRB10, H19, IGF2, CDKN1C, WT1, TCEB3C, PEG3, GNAS, ZNF331). Cancer was the single disease category (1.43-fold enrichment, $\mathrm{p}=3.42 \times 10^{-5}$ ) significantly over-represented based on the presence of 96 genes associated with its etiology or progression (Additional File 3, Table S3). Clustering of those 1,423 genes based on ontology resulted in 14 significantly over-represented functional categories (Table 2; Additional File 4, Table S4). The principal functions represented by these categories were mesodermal development, metabolic regulation, nucleocytoplasmic transport, and transcriptional regulation.

\section{Relationship between maternal age and newborn DNA methylation in blood cell type signature genes}

Of 852 loci with official gene symbols whose expression levels are signatures of blood cell types [38], we found 658 that were assayed by the Humanmethylation 27 array. These 658 genes are represented by $1,273 \mathrm{CpG}$ probes on the Illumina array (Additional File 5, Table S5). The first principal component based on the methylation levels of these probes among the 168 newborns explained $97.9 \%$ of the variation. Both visually and based on Spearman correlation ( $\mathrm{rho}=0.07, \mathrm{p}=0.34$ ), there was no apparent relationship between the principal component representing combined blood cell signature gene methylation and maternal age. Of those 1,273 individual probes, 7 (0.55\%; within genes C19orf6, GM2A, SEPX1, RPL27, CTSZ, PGAM1, PLXDC1) were among those significantly correlated with maternal age. Given that this proportion is nearly identical to the proportion of CpG probes genome-wide showing significant correlation with maternal age $(0.52 \%$; $\mathrm{P}(7 / 1,273 \geq 0.52 \%)=$ 0.49 , binomial test), blood cell type signature genes do not appear to be enriched among the significant CpG probes, thereby providing indirect evidence against changes in umbilical blood cell composition with maternal age.

\section{Discussion}

To the extent of our knowledge, this is the first demonstration of a trans-generational effect of parental, primarily maternal, age on the levels of DNA methylation in the next generation at birth. Clusters of CpG dinucleotides, or CpG islands, are disproportionately located in the upstream, regulatory regions of genes and typically exhibit lower levels of methylation than do nonisland CpGs. In this sense the trend we observed towards decreased methylation of newborn CpG islands with increasing parental age represents an accentuation of a normal pattern of DNA methylation. In this context, the question becomes whether these changes are related to disease or other phenotypic outcomes in the offspring. As was noted earlier, the children of older parents have increased risks of type 1 diabetes, neurodevelopmental disorders, and cancer. Therefore, it is noteworthy that cancer was the disease category over-represented by genes whose methylation strongly correlated with maternal age. A survey of Online Mendelian Inheritance in Man (OMIM) [39] for the 142 genes that achieved genome-wide significance with age in our sample reveals several genes with known functional or molecular associations with cancer, obesity, and diabetes (Table 3). This is suggestive of a possible role of maternal age-associated shifts in newborn methylation in the increased risks of these outcomes. However,

Table 2 Functional clusters of 1,493 CpGs (1,423 genes) with strongest correlations between levels of methylation and maternal age according to DAVID

\begin{tabular}{|c|c|c|c|}
\hline Terms & \# Genes & Minimum DAVID $p$ Value & Enrichment Score \\
\hline Mesenchymal cell development/differentiation & 11 & 0.003 & 2.53 \\
\hline Formation of mesoderm/primary germ layer & 9 & 0.003 & 2.34 \\
\hline Neurological regulation & 19 & 0.008 & 1.83 \\
\hline Regulation of glucose/carbohydrate metabolic process & 8 & 0.01 & 1.83 \\
\hline Unsaturated fatty/icosanoid metabolism/biosynthesis & 8 & 0.005 & 1.71 \\
\hline Negative regulation nucleocytoplasmic protein/transcription factor transport & 5 & 0.006 & 1.70 \\
\hline Regulation nucleocytoplasmic protein/transcription factor transport & 10 & 0.01 & 1.60 \\
\hline Limb morphogenesis & 14 & 0.02 & 1.57 \\
\hline Phosphopantetheine-binding, acyl carrier activity & 3 & 0.01 & 1.53 \\
\hline MADS-box & 3 & 0.04 & 1.40 \\
\hline Helix-loop-helix & 14 & 0.03 & 1.37 \\
\hline Positive regulation of glucose/carbohydrate metabolism & 5 & 0.04 & 1.33 \\
\hline Negative regulation of phosphorylation & 8 & 0.04 & 1.33 \\
\hline Regulation of transmembrane transport & 6 & 0.03 & 1.32 \\
\hline
\end{tabular}


Table 3 Diseases and functions reported to be associated with genes exhibiting a significant correlation between methylation and maternal age

\begin{tabular}{|c|c|c|}
\hline Gene & Phenotype/Function & OMIM Reference \\
\hline KCNQ1 & $\begin{array}{c}\text { Long QT Syndrome 1; Jervell and Lange-Nielsen Syndrome 1; Atrial fibrillation, familial 3; short QT } \\
\text { Syndrome }\end{array}$ & $\begin{array}{l}192500220400607554 \\
609621\end{array}$ \\
\hline TUBB2A & Polymicrogyria, asymmetric & 610031 \\
\hline RAD51 & Breast cancer, familial & 114480 \\
\hline C19orf6 & Ovarian, breast, pancreatic and colorectal cancer expression & 611011 \\
\hline RAD54L & Adenocarcinoma, colonic, somatic; Lymphoma, non-Hodgkin; Breast cancer, invasive ductal & 603615 \\
\hline ACADS & SCAD deficiency & 201470 \\
\hline GM2A & Gangliosidase, $A B$ variant & 272750 \\
\hline STAT5B & Growth hormone insensitivity with immunodeficiency & 245590 \\
\hline RET & $\begin{array}{c}\text { Multiple endocrine neoplasia, type IIA; Hirschprung disease; Central hypoventilation syndrome; renal } \\
\text { agenesis }\end{array}$ & 142623209880191830 \\
\hline ZFYVE26 & Spastic paraplegia 15 , autosomal recessive & 270700 \\
\hline RBBP9 & Transformation to cancer & 602908 \\
\hline MRPS22 & Combined oxidative phosphorylation deficiency 5 & 611719 \\
\hline MYC & Burkitt lymphoma & 113970 \\
\hline RFXDC1 & Diabetes, neonatal, with pancreatic hypoplasia, intestinal atresia and gallbladder aplasia or hypoplasia & 601346 \\
\hline MYST1 & Histone acetyltransferase & 609912 \\
\hline RASSF1 & Tumor suppressor & 605082 \\
\hline ZBT16 & Skeletal defects, genital hypoplasia, and mental retardation & 612447 \\
\hline ACTN4 & Focal glomerulosclerosis 1 & 603278 \\
\hline NROB2 & Obesity, mild, early-onset & 604630 \\
\hline ALX4 & Parietal foramina 2 & 609597 \\
\hline
\end{tabular}

it must be emphasized that the associations listed in OMIM are almost exclusively due to genetic defects in these genes, while hypomethylation of CpGs, particularly in CpG islands, is expected to result in increased expression of these genes, rather than repression.

According to DAVID $[40,41]$, there were 14 functions enriched among the 1,493 genes with the strongest correlations between maternal age and newborn DNA methylation. The category with the second greatest enrichment was mesodermal development, and this is not surprising given that white blood cells originate from the mesodermal layer. The third greatest enrichment was for functions related to neurological regulation, including strong candidate genes for psychiatric disorders such as SLC6A1, GNAI3, PTGS2, and CTNND2. Given the association between increasing parental age and schizophrenia, bipolar disorder and autism in offspring, studies of shifts in the methylation of these genes in offspring of older parents are warranted. Similarly, the risk of diabetes appears to be elevated in the children of older parents, and functions related to glucose and carbohydrate metabolism are enriched among the genes whose methylation is correlated with maternal age, including such candidate genes for obesity and diabetes as IRS1, IRS2, DUSP12, $D Y R K 2$, and ARNT.
Multiple reports have documented significant changes in DNA methylation patterns with age in adults, both as a genome-wide average and at specific nucleotide sites. The question arises as to whether these adult patterns mirror those we observed in newborns, which would imply that the same processes are operating both in adult somatic tissues and in either gametogenesis or embryonic development. Of the 589 CpGs with significant correlation with age in adults discovered by Teschendorff et al. [13], only one (in PLAT) achieved genome-wide significance among our newborns. Similarly, of the 131 CpGs studied by Rakyan et al. [11] whose methylation levels in adults were positively correlated with age and were highly replicable, only one (in RFXDC1) was observed by us to have a trans-generational effect. By contrast, 30 genes overlapped between Rakyan et al. and Teschendorff et al. Among the 288 CpGs studied by Boks et al. [10], 90 loci located in 69 genes were associated with adult age at $\mathrm{p} \leq 0.05$. CpGs in two of those genes (GAS7 and $R Y K$ ) were also significant in our cohort. Likewise, of the $106 \mathrm{CpGs}$ in 73 genes found to be significantly related to age in blood by Christensen et al. [9], only one gene (PLAT) was among our significant results. With the exception of a very small number of genes, these comparisons indicate that the processes resulting in age-related trends in 
DNA methylation in adult somatic tissues and in newborn somatic tissues may be independent.

There are two most likely possibilities to explain the differences. First, the patterns of change in methylation in oocytes and/or spermatocytes with respect to age are different than in somatic tissue and these are reflected in shifted patterns in the newborn. This explanation can be tested by comparison of methylation patterns in somatic tissues and gametes in the same individuals with respect to age. Second, the mechanisms of establishment and/or maintenance of DNA methylation in the developing fetus could be responsive to parental age, but in a manner different than in the adult somatic tissues. This hypothesis cannot be tested by epidemiological methods but instead requires detailed molecular biological examination.

It is well-established that DNA methylation of imprinted genes is established in a parent of origin manner during development of the germ line and gametogenesis [42], while methylation of other loci is thought to be re-established after a nearly global demethylation subsequent to fertilization and uterine implantation. Given the stringency with which DNA methylation is thought to be maintained at imprinted loci, it is somewhat surprising that DNA methylation at 2 imprinted loci exhibited genomewide-wide significant correlations with maternal age and a total of 12 met the less stringent q value threshold of $6.69 \times 10^{-4}$. However, there is evidence that the methylation patterns present in the parents are not totally erased and reset during gametogenesis and fertilization at a proportion of loci, including both imprinted and non-imprinted genes. Borgel et al. [43] identified a set of 215 genes that are methylated in the mouse very early in embryonic development and well before implantation. Of the few loci tested, all were methylated in gametes, with some shifts in those patterns being observed in preimplantation embryos. Although these observations do not address the issue of whether age-related changes in DNA methylation previously found in somatic tissues are mechanistically related to the trends we have tentatively identified to be related to maternal age, they do indicate a definite potential for trans-generational inheritance of DNA methylation patterns

A proportion of the age-related changes in DNA methylation observed in adult cohorts has been attributed to changes in blood cell composition as an individual ages. Therefore, the possibility exists that the changes in patterns of newborn DNA methylation which were observed may be at least partly due to changes in umbilical cord blood cell composition during pregnancy. However, we found no significant relationship between gestational age and CpG methylation in newborn cord blood, and therefore the gestational age of the fetus is an unlikely explanation for these differences. There is no obvious mechanism by which the mother's (or father's) age would shift cord blood cell composition. We did not directly assess the relative proportion of cell types in our cord blood samples. In our examination of the methylation patterns among genes whose expression levels are characteristic of major blood cell types, we found no obvious relationship to maternal age or over-representation of these genes among the significant results. Although this does not disprove that changes in blood cell composition explain all or part of our results, these findings are not consistent with substantial differences in blood cell composition or the regulation via methylation of the genes that are signatures of major cell subtypes.

There is the obvious question as to whether the changes in DNA methylation patterns we observed in umbilical cord blood are representative of patterns in other tissues of the newborn. It is widely accepted that diverging patterns of methylation at individual loci are a key aspect of cellular differentiation during development, and as a consequence levels of methylation at a subset of loci between two different tissue types will differ. However, there is also evidence of similarities in patterns of methylation across tissue types. For example, methylation levels of IGF2 and $E R-\alpha$ in peripheral blood mirror those of colon tissue $[44,45]$. Additionally, comparisons among multiple tissues from multiple autopsy subjects reveal individualspecific and locus-specific patterns of DNA methylation but a high degree of similarity among tissues within the same individual [46]. Likewise, within an individual there is similarity in methylation patterns between blood and buccal cells [47]. Given that these similarities are shared among tissues with different embryonic origins, this suggests that the similarities are established early in development and are fairly persistent throughout life.

\section{Conclusions}

At a subset of loci, levels of DNA methylation in umbilical cord blood are strongly correlated with maternal, and to a lesser extent paternal, age. This correlation is predominantly negative and disproportionately occurs within CpG islands. Genes associated with oncogenesis and cancer progression are significantly over-represented among the genes correlated with maternal age, and this suggests a link to known increased risks of cancer among the children of older parents. Similarly, gene functions related to neurodevelopment and neuroregulation are over-represented among the strongly correlated genes, and this may have relevance to the increasing risk of neurodevelopmental and psychiatric disorders in offspring as parental ages increase. 


\section{Methods}

\section{Participants and Samples}

From a longitudinal study (Conditions Affecting Neurocognitive Development and Learning in Early Childhood; CANDLE) of child development from the second trimester of a singleton pregnancy until age 3 in Memphis TN, the first 168 newborns were chosen for which blood samples were available for both the mother and her newborn and for which there were no infectious or chronic illnesses in the mothers and no pregnancy complications. Newborn blood was collected from the umbilical cord at delivery. After centrifugation of whole blood, the buffy coat was collected and frozen. Subsequently, Wizard genomic DNA purification reagents (Promega Corp.) were used for DNA extraction. All demographic and phenotypic data on the mothers and newborns were abstracted from clinical records. Paternal ages were reported by the mothers via mail questionnaires and phone calls during the months after delivery of the newborn. This research was approved by the Institutional Review Board of the University of Tennessee Health Science Center, and informed consent was obtained from all mothers.

\section{Genome-wide measurement of DNA methylation levels}

Bisulfite conversion of $750 \mathrm{ng}$ of genomic DNA was performed using EZ DNA Methylation reagents (Zymo Research). Samples were then processed according to manufacturer's specifications and hybridized and scanned on the Humanmethylation27 BeadChip (Illumina Inc.) in batches of 24 samples using the Illumina BeadStation. The Humanmethylation27 BeadChip bears probes for 27,578 specific CpG dinucleotides assigned to 14,495 loci. The level of methylation of each CpG is represented by a beta value, which is calculated as the level of the fluorescence for the probe specific for 5methylcytosine divided by the fluorescence from the probes for both the methylated and unmethylated $\mathrm{C}$ at that position. Consequently, the beta values span the bounded range 0 - 1 . The data has been submitted to the Gene Expression omnibus data base with accession number GSE27317.

Subsequent to statistical analyses, three CpGs with differing levels of methylation from the Illumina data that also were among the top-ranked p values were chosen for validation by pyrosequencing. This work was performed by a commercial service (EpigenDx, Worcester, MA). Upon receipt of 92 DNA samples, EpigenDx performed the bisulfite conversion, designed the assays, and performed pyrosequencing with the inclusion of high and low methylation controls. For all three assays, results from control DNAs indicated complete bisulfite conversion.

\section{Correlation between newborn CpG methylation and both parental and newborn characteristics}

Raw output files from array hybridization experiments were processed using GenomeStudio software (Illumina Inc.). This software reports detection $\mathrm{p}$ values for each CpG interrogated by the array, which are an indication of the ability to distinguish the target sequence from background. Beta values representing the proportion of methylation at each site and the corresponding detection $\mathrm{p}$ values were imported into Microsoft SQL Server 2005, where filtering of the data values was performed before statistical analyses. For each newborn, probes with detection $\mathrm{p}$ values $\geq 10^{-3}$ were dropped from that individual. Additionally, any probe with a median detection $\mathrm{p}$ value $\geq 10^{-6}$ across newborns was dropped from all individuals.

Statistical analyses were performed using SPSS version 18 (SPSS Inc.) and Stata 10 (Stata Corp.). Several variables related to the mothers, fathers and newborns could possibly be related to DNA methylation levels at individual CpG sites. Therefore, we performed statistical analyses to examine the association between each of these variables and the level of methylation at each CpG. Because the distribution of DNA methylation at many sites is not normal, we performed nonparametric analyses. Specifically, for continuous variables (maternal age, paternal age, parity, maternal body mass index, gestational age, birth weight) we performed Spearman rank correlation, and for binary variables (maternal smoking during pregnancy, newborn gender) we performed Wilcoxon rank sum analysis. Maternal BMI was based on the mother's height and her report of prepregnancy weight at the time of recruitment in the early second trimester of pregnancy. Prior to analysis, regression of birth weight on gestational age, gender, maternal prepregnancy $\mathrm{BMI}$ and race was performed, and the residuals used for correlation. A Bonferroni-corrected genome-wide threshold for statistical significance of $1.81 \times 10^{-6}$ for the relationship between each variable and the levels of DNA methylation at each CpG was employed based on the 27,576 tests performed for each variable.

Systematic differences between the two races in DNA methylation levels or maternal age are potential confounders in the analyses of correlations between maternal age and newborn DNA methylation. To address the influence of these potential confounders, we used independent genome-wide SNP data collected on a subset (63 African-American, 68 Caucasian) of the participants using the Affymetrix (Santa Clara CA, USA) Genomewide SNP 5.0 and 6.0 arrays. Samples were processed according to Affymetrix's protocol and genotypes were called using the BRLMM (5.0 array) and Birdseed (6.0 
array) algorithms with default parameters within the Affymetrix Genotyping Console v4.0 application. Based on the genome-wide patterns of SNP genotypes, loadings on the first two principal components were calculated using the SNP and Variation Suite module of the GoldenHelix v7 software (Golden Helix Inc., Bozeman MT, USA). Based on plots of the loadings on the first two principal components, two genetically distinct groups of participants were identified. Within these comparatively more genetically homogeneous groups, correlations between maternal age and newborn DNA methylation were performed for comparison to analyses across all individuals to determine if population substructuring of genetic variation confounded the main analyses of parental age effects on DNA methylation patterns.

\section{Functional and disease clustering of significant genes}

The Database for Annotation, Visualization, and Integrated Discovery [DAVID, 41, 48] was used to group genes according to functional category and to identify disease processes highly represented among the loci most strongly associated with maternal age. The Bonferroni correction we used to assign significance to loci is likely to be too stringent and to exclude many loci truly correlated with maternal age. Therefore, for the purpose of identifying potentially affected physiological functions and disease processes, we based our selection of loci on false discovery rate q values, or the expected proportion of loci at that threshold of significance that are likely to be false positives. A visual inspection of plots of the number of tests declared significant versus the q value produced by the QVALUE program $[49,50]$ illustrated that the relationship sharply changed beyond a $q$ value of about 0.001 . To be conservative, we used a q value threshold of $6.69 \times 10^{-4}$ (corresponding to a $\mathrm{p}$ value of $\left.9.55 \times 10^{-5}\right)$, which results in an expectation that approximately one CpG out of the 1,493 declared significant is a false positive. These 1,493 CpGs belonged to 1,423 different genes. For identification of functional clusters, we used the highest classification stringency and restricted attention to enrichment scores $\geq 1.3$, roughly corresponding to a $\mathrm{p}$ value of $0.05\left(10^{-1.3}\right)$. As the background reference set of genes, we used the nonredundant list of genes represented on the Humanmethylation 27 array as identified by the Entrez gene ID numbers provided by Illumina in their annotation of the array. The identification of imprinted loci was based on the online imprinted gene and parent-of-origin effect database http://igc.otago.ac.nz/home.html [51]. Using liberal criteria, we considered 59 loci with known or polymorphic imprinting patterns or provisional evidence of imprinting.
Univariate and multivariate analyses of genes whose expression or DNA methylation are characteristic of blood cell types

Previous observations of a relationship between adult age and changes in DNA methylation have sometimes been attributed to changes in blood cell composition as an individual ages. Palmer et al. [38] identified genes whose expression is characteristic of B-cells, T-cell subtypes, granulocytes and lymphocytes. Although they did not directly determine the blood cell type composition of their ovarian cancer case-control cohort, Teschendorff et al. [52] used Palmer et al.'s [38] blood cell subtype-specific expression signatures and the same methylation array used in our study to infer that agerelated changes in DNA methylation in their cohort could principally be explained by changes in blood cell composition based on the over-representation of genes whose expression levels are signatures of blood cell subtypes. To indirectly address the possibility that the patterns we observe could be due to maternal age-related changes in the relative proportions of newborn cord blood cell types, we also used the data of Palmer et al. [38].

Based on gene symbols, we identified the overlap between the signature genes reported by Palmer et al. and the genes targeted by the Illumina methylation array. Using all of the probes among these genes, we performed principal components analysis. To visually examine the relationship, we plotted the first principal component versus maternal age. Additionally, we calculated the Spearman rank correlation between maternal age and the first principal component. The principal component analysis summarizes the variability due to all of the probes in the blood cell type signature genes. However, any relationship between maternal age and changes in cord blood cell type composition may be due to a subset of genes or probes. Therefore, we also examined the rank correlation between maternal age and each probe in signature genes.

\section{Additional material}

Additional file 1: Table S1. Summary of statistical results for each CpG, including select annotation from Illumina.

Additional file 2: Table S2. List of the 144 CpGs with genome-wide significant correlation between newborn methylation and maternal age and the CpG with next smallest $p$ value. Whether the $1^{\text {st }}$ and $2^{\text {nd }} \mathrm{CpG}$ exhibit correlation with maternal age in the same direction and whether the $2^{\text {nd }} \mathrm{CpG}$ is significant at a nominal $p$ value of 0.05 is indicated.

Additional file 3: Table S3. Output from DAVID analysis of disease clustering among the 1,423 genes whose level of methylation was correlated with maternal age at a q value of $6.69 \times 10^{-4}$, corresponding to an expectation of one false positive and $p=9.55 \times 10^{-5}$.

Additional file 4: Table S4. Output of functional clustering of 1,423 genes whose level of newborn methylation was correlated with maternal 
age at a $q$ value of $6.69 \times 10^{-4}$, corresponding to an expectation of one false positive and $p=9.55 \times 10^{-5}$ by DAVID.

Additional file 5: Table S5. List of 1,273 Humanmethylation27 array probes and 658 genes overlapping with blood cell type signature genes identified by Palmer et al. (2006. Cell-type specific gene expression profiles of leukocytes in human peripheral blood. BMC Genomics 7, 115).

\section{List of abbreviations}

DAVID: Database for Annotation, Visualization, and Integrated Discovery; DMR: differentially methylated region; OMIM: Online Mendelian Inheritance in Man; CANDLE: Conditions Affecting Neurocognitive Development and Learning in Early Childhood;

\section{Acknowledgements}

This project was supported by grant HD060713 to RMA from the National Institute of Child Health and Human Development. Its contents are solely the responsibility of the authors and do not necessarily represent the official views of the NICHD. Additional support came from a grant from the University of Tennessee Health Science Center's Clinical and Translational Science Institute to RMA and a grant from The Urban Child Institute to FT. None of the funding sources had any role in the design, implementation or interpretation of this work or in the manuscript preparation. We gratefully acknowledge insightful conversations with Dr. Vicki M. Park, the laboratory expertise of Jeanette Peeples and Joycelynn Butler, the analytical and data management skills of Priyanka Jani and Yanhua Qu, the participant recruitment and sample collection by CANDLE staff, and particularly the mothers who consented to participate.

\section{Author details}

${ }^{1}$ Department of Pediatrics, University of Tennessee Health Science Center, Memphis, TN 38103, USA. ${ }^{2}$ Department of Preventive Medicine, University of Tennessee Health Science Center, Memphis, TN 38163, USA.

\section{Authors' contributions}

RMA designed the project and directed the collection and analyses of experimental data. FAT assisted in the interpretation of the data and directs the CANDLE project, its experimental design, and collection of biological samples. FT and JK participated in the analyses and interpretation of the data. All authors read and approved the final manuscript.

Received: 2 August 2010 Accepted: 31 March 2011 Published: 31 March 2011

\section{References}

1. Eckhardt F, Lewin J, Cortese R, Rakyan VK, Attwood J, Burger M, Burton J, Cox TV, Davies R, Down TA, Haefliger C, Horton R, Howe K, Jackson DK, Kunde J, Koenig C, Liddle J, Niblett D, Otto T, Pettett R, Seemann S, Thompson C, West T, Rogers J, Olek A, Berlin K, Beck S: DNA methylation profiling of human chromosomes 6, 20 and 22. Nat Genet 2006 38(12):1378-1385.

2. Rakyan VK, Hildmann T, Novik KL, Lewin J, Tost J, Cox AV, Andrews TD, Howe KL, Otto T, Olek A, Fischer J, Gut IG, Berlin K, Beck S: DNA methylation profiling of the human major histocompatibility complex: a pilot study for the human epigenome project. PLOS Biol 2004, 2(12):e405.

3. Song F, Smith JF, Kimura MT, Morrow AD, Matsuyama T, Nagase H, Held WA: Association of tissue-specific differentially methylated regions (TDMs) with differential gene expression. Proc Natl Acad Sci USA 2005, 102(9):3336-3341.

4. Weaver JR, Susiarjo M, Bartolomei MS: Imprinting and epigenetic changes in the early embryo. Mamm Genome 2009, 20(9-10):532-543.

5. Wilson AS, Power BE, Molloy PL: DNA hypomethylation and human diseases. Biochim Biophys Acta 2007, 1775(1):138-162.

6. Gravina S, Vijg J: Epigenetic factors in aging and longevity. Pflugers Arch 2010, 459(2):247-258

7. Fuke C, Shimabukuro M, Petronis A, Sugimoto J, Oda T, Miura K, Miyazaki T, Ogura C, Okazaki Y, Jinno Y: Age related changes in 5-methylcytosine content in human peripheral leukocytes and placentas: an HPLC-based study. Ann Hum Genet 2004, 68(Pt 3):196-204.
8. Fraga MF, Ballestar E, Paz MF, Ropero S, Setien F, Ballestar ML, HeineSuner D, Cigudosa JC, Urioste M, Benitez J, Boix-Chornet M, SanchezAguilera A, Ling C, Carlsson E, Poulsen P, Vaag A, Stephan Z, Spector TD, Wu YZ, Plass C, Esteller M: Epigenetic differences arise during the lifetime of monozygotic twins. Proc Natl Acad Sci USA 2005, 102(30):10604-10609.

9. Christensen BC, Houseman EA, Marsit CJ, Zheng S, Wrensch MR, Wiemels JL, Nelson HH, Karagas MR, Padbury JF, Bueno R, Sugarbaker DJ, Yeh RF, Wiencke JK, Kelsey KT: Aging and environmental exposures alter tissue-specific DNA methylation dependent upon $\mathrm{CpG}$ island context. PLoS Genet 2009, 5(8):e1000602.

10. Boks MP, Derks EM, Weisenberger DJ, Strengman E, Janson E, Sommer IE, Kahn RS, Ophoff RA: The relationship of DNA methylation with age, gender and genotype in twins and healthy controls. PLoS One 2009, 4(8): e6767.

11. Rakyan VK, Down TA, Maslau S, Andrew T, Yang TP, Beyan H, Whittaker P, McCann OT, Finer S, Valdes AM, Leslie RD, Deloukas P, Spector TD: Human aging-associated DNA hypermethylation occurs preferentially at bivalent chromatin domains. Genome Res 2010, 20(4):434-439.

12. Liu J, Morgan M, Hutchison K, Calhoun VD: A study of the influence of sex on genome wide methylation. PLoS One 2010, 5(4):e10028.

13. Teschendorff AE, Menon U, Gentry-Maharaj A, Ramus SJ, Weisenberger DJ, Shen H, Campan M, Noushmehr H, Bell CG, Maxwell AP, Savage DA, Mueller-Holzner E, Marth C, Kocjan G, Gayther SA, Jones A, Beck S, Wagner W, Laird PW, Jacobs IJ, Widschwendter M: Age-dependent DNA methylation of genes that are suppressed in stem cells is a hallmark of cancer. Genome Res 2010, 20(4):440-446.

14. Bollati V, Schwartz J, Wright R, Litonjua A, Tarantini L, Suh H, Sparrow D, Vokonas $\mathrm{P}$, Baccarelli $\mathrm{A}$ : Decline in genomic DNA methylation through aging in a cohort of elderly subjects. Mech Ageing Dev 2009, 130(4):234-239.

15. Bjornsson HT, Sigurdsson MI, Fallin MD, Irizarry RA, Aspelund T, Cui $H, Y u$ W, Rongione MA, Ekstrom TJ, Harris TB, Launer LJ, Eiriksdottir G, Leppert MF, Sapienza C, Gudnason V, Feinberg AP: Intra-individual change over time in DNA methylation with familial clustering. JAMA 2008, 299(24):2877-2883.

16. Sartorius GA, Nieschlag E: Paternal age and reproduction. Hum Reprod Update 2010, 16(1):65-79.

17. Cardwell CR, Carson DJ, Patterson CC: Parental age at delivery, birth order, birth weight and gestational age are associated with the risk of childhood Type 1 diabetes: a UK regional retrospective cohort study. Diabet Med 2005, 22(2):200-206

18. King MD, Fountain C, Dakhlallah D, Bearman PS: Estimated autism risk and older reproductive age. Am J Public Health 2009, 99(9):1673-1679.

19. Sipos A, Rasmussen F, Harrison G, Tynelius P, Lewis G, Leon DA, Gunnell D: Paternal age and schizophrenia: a population based cohort study. BMJ 2004, 329(7474):1070.

20. Menezes PR, Lewis G, Rasmussen F, Zammit S, Sipos A, Harrison GL, Tynelius P, Gunnell D: Paternal and maternal ages at conception and risk of bipolar affective disorder in their offspring. Psychol Med 2010, 40(3):477-485.

21. Comings DE, MacMurray JP: Maternal age at the birth of the first child as an epistatic factor in polygenic disorders. American Journal of Medical Genetics Part B: Neuropsychiatric Genetics 2006, 141B(1):1-6.

22. Lu Y, Ma H, Sullivan-Halley J, Henderson KD, Chang ET, Clarke CA, Neuhausen SL, West DW, Bernstein L, Wang SS: Parents' Ages at Birth and Risk of Adult-onset Hematologic Malignancies Among Female Teachers in California. Am J Epidemiol 2010, 171(12):1262-1269.

23. Johnson KJ, Carozza SE, Chow EJ, Fox EE, Horel S, McLaughlin CC, Mueller BA, Puumala SE, Reynolds P, Von Behren J, Spector LG: Parental age and risk of childhood cancer: a pooled analysis. Epidemiology 2009, 20(4):475-483.

24. Podvin D, Kuehn CM, Mueller BA, Williams M: Maternal and birth characteristics in relation to childhood leukaemia. Paediatr Perinat Epidemiol 2006, 20(4):312-322.

25. Maule MM, Vizzini L, Merletti F, Magnani C, Pastore G, Richiardi L: Parental age and risk of acute lymphocytic leukaemia and embryonal tumours in the Piedmont Region, Italy. Int J Epidemio/ 2007, 36(3):691-692.

26. Maule MM, Merletti F, Pastore G, Magnani C, Richiardi L: Effects of maternal age and cohort of birth on incidence time trends of childhood acute lymphoblastic leukemia. Cancer Epidemiol Biomarkers Prev 2007, 16(2):347-351. 
27. Yip BH, Pawitan Y, Czene K: Parental age and risk of childhood cancers: a population-based cohort study from Sweden. Int J Epidemiol 2006, 35(6):1495-1503

28. Hemminki K, Kyyronen P, Vaittinen P: Parental age as a risk factor of childhood leukemia and brain cancer in offspring. Epidemiology 1999, 10(3):271-275.

29. Moll AC, Imhof SM, Kuik DJ, Bouter LM, Den Otter W, Bezemer PD, Koten JW, Tan KE: High parental age is associated with sporadic hereditary retinoblastoma: the Dutch retinoblastoma register 1862-1994. Hum Genet 1996, 98(1):109-112.

30. Hodgson ME, Newman B, Millikan RC: Birthweight, parental age, birth order and breast cancer risk in African-American and white women: a population-based case-control study. Breast Cancer Res 2004, 6(6): R656-667.

31. Zhang Y, Kreger BE, Dorgan JF, Cupples LA, Myers RH, Splansky GL, Schatzkin A, Ellison RC: Parental age at child's birth and son's risk of prostate cancer. The Framingham Study. Am J Epidemiol 1999, 150(11):1208-1212.

32. Dolinoy DC, Weidman JR, Waterland RA, Jirtle RL: Maternal genistein alters coat color and protects Avy mouse offspring from obesity by modifying the fetal epigenome. Environ Health Perspect 2006, 114(4):567-572.

33. Li E: Chromatin modification and epigenetic reprogramming in mammalian development. Nat Rev Genet 2002, 3(9):662-673.

34. Reik W, Dean W, Walter J: Epigenetic reprogramming in mammalian development. Science 2001, 293(5532):1089-1093.

35. Cotton AM, Avila L, Penaherrera MS, Affleck JG, Robinson WP, Brown CJ: Inactive $\mathrm{X}$ chromosome-specific reduction in placental DNA methylation. Hum Mol Genet 2009, 18(19):3544-3552.

36. Philibert RA, Sandhu H, Hollenbeck N, Gunter T, Adams W, Madan A: The relationship of $5 \mathrm{HTT}$ (SLC6A4) methylation and genotype on mRNA expression and liability to major depression and alcohol dependence in subjects from the lowa Adoption Studies. Am J Med Genet B Neuropsychiatr Genet 2008, 147B(5):543-549.

37. Adkins RM, Krushkal J, Tylavsky F, Thomas F: Racial Differences in GeneSpecific DNA Methylation Levels are Present at Birth. Birth Defects Res A Clin Mol Teratol 2011.

38. Palmer C, Diehn M, Alizadeh AA, Brown PO: Cell-type specific gene expression profiles of leukocytes in human peripheral blood. BMC Genomics 2006, 7:115.

39. Online Mendelian Inheritance in Man. [http://www.ncbi.nlm.nih.gov/ omim/].

40. Database for Annotation, Visualization and Integrated Discovery (DAVID). [http://david.abcc.ncifcrf.gov/].

41. Dennis G Jr, Sherman BT, Hosack DA, Yang J, Gao W, Lane HC, Lempicki RA DAVID: Database for Annotation, Visualization, and Integrated Discovery. Genome Biol 2003, 4(5):P3.

42. Tucker KL, Beard C, Dausmann J, Jackson-Grusby L, Laird PW, Lei H, Li E, Jaenisch R: Germ-line passage is required for establishment of methylation and expression patterns of imprinted but not of nonimprinted genes. Genes Dev 1996, 10(8):1008-1020.

43. Borgel J, Guibert S, Li Y, Chiba H, Schubeler D, Sasaki H, Forne T, Weber M: Targets and dynamics of promoter DNA methylation during early mouse development. Nat Genet 2010, 42(12):1093-1100.

44. Cui H, Cruz-Correa M, Giardiello FM, Hutcheon DF, Kafonek DR, Brandenburg S, Wu Y, He X, Powe NR, Feinberg AP: Loss of IGF2 imprinting: a potential marker of colorectal cancer risk. Science 2003, 299(5613):1753-1755.

45. Ally MS, Al-Ghnaniem R, Pufulete M: The relationship between genespecific DNA methylation in leukocytes and normal colorectal mucosa in subjects with and without colorectal tumors. Cancer Epidemiol Biomarkers Prev 2009, 18(3):922-928.

46. Byun HM, Siegmund KD, Pan F, Weisenberger DJ, Kanel G, Laird PW, Yang AS: Epigenetic profiling of somatic tissues from human autopsy specimens identifies tissue- and individual-specific DNA methylation patterns. Hum Mol Genet 2009, 18(24):4808-4817.

47. Talens RP, Boomsma DI, Tobi EW, Kremer D, Jukema JW, Willemsen G, Putter H, Slagboom PE, Heijmans BT: Variation, patterns, and temporal stability of DNA methylation: considerations for epigenetic epidemiology. FASEB J 2010, 24(9):3135-3144.
48. Huang da W, Sherman BT, Lempicki RA: Systematic and integrative analysis of large gene lists using DAVID bioinformatics resources. Nat Protoc 2009, 4(1):44-57.

49. Storey JD, Tibshirani R: Statistical significance for genomewide studies. Proc Natl Acad Sci USA 2003, 100(16):9440-9445.

50. QVALUE software. [http://www.genomine.org/qvalue/].

51. Morison IM, Paton CJ, Cleverley SD: The imprinted gene and parent-oforigin effect database. Nucleic Acids Res 2001, 29(1):275-276.

52. Teschendorff AE, Menon U, Gentry-Maharaj A, Ramus SJ, Gayther SA, Apostolidou S, Jones A, Lechner M, Beck S, Jacobs IJ, Widschwendter M: An epigenetic signature in peripheral blood predicts active ovarian cancer. PloS One 2009, 4(12):e8274.

\section{Pre-publication history}

The pre-publication history for this paper can be accessed here: http://www.biomedcentral.com/1471-2350/12/47/prepub

doi:10.1186/1471-2350-12-47

Cite this article as: Adkins et al:: Parental ages and levels of DNA methylation in the newborn are correlated. BMC Medical Genetics 2011 12:47.

\section{Submit your next manuscript to BioMed Central and take full advantage of:}

- Convenient online submission

- Thorough peer review

- No space constraints or color figure charges

- Immediate publication on acceptance

- Inclusion in PubMed, CAS, Scopus and Google Scholar

- Research which is freely available for redistribution

Submit your manuscript at www.biomedcentral.com/submit 